\title{
New concept of relativistic invariance in NC space-time: twisted Poincaré symmetry and its implications
}

\author{
M. Chaichian, P. Prešnajder ${ }^{1}$ and A. Tureanu \\ High Energy Physics Division, Department of Physical Sciences, \\ University of Helsinki, and Helsinki Institute of Physics \\ P.O. Box 64, FIN-00014 Helsinki, Finland
}

\begin{abstract}
We present a systematic framework for noncommutative (NC) QFT within the new concept of relativistic invariance based on the notion of twisted Poincaré symmetry (with all 10 generators), as proposed in ref. [7]. This allows to formulate and investigate all fundamental issues of relativistic QFT and offers a firm frame for the classification of particles according to the representation theory of the twisted Poincaré symmetry and as a result for the $\mathrm{NC}$ versions of CPT and spin-statistics theorems, among others, discussed earlier in the literature. As a further application of this new concept of relativism we prove the $\mathrm{NC}$ analog of Haag's theorem.
\end{abstract}

\footnotetext{
${ }^{1}$ On leave of absence from the Department of Theoretical Physics and Physics Teaching, Comenius University, Mlynská dolina, SK-84248 Bratislava, Slovakia
} 


\section{Introduction}

The idea that coordinates may not commute can be traced back to Heisenberg. For an early reference on field theory in a noncommutative space-time, see [1]. The noncommutative (NC) QFT in Moyal space-time was formulated in [2]. Within this approach the fields are functions of $\mathrm{NC}$ coordinates $\hat{x}^{\mu}$, $\mu=0,1,2,3$, satisfying the commutation relations

$$
\left[\hat{x}^{\mu}, \hat{x}^{\nu}\right]=\mathrm{i} \theta^{\mu \nu}, \theta^{\mu \nu}=-\theta^{\nu \mu}-\text { real constants } .
$$

This type of theory emerges also as a low energy limit of the string theory in a particular background, [3]. It is well-known that if there is space-time noncommutativity, i.e. some of $\theta^{0 i} \neq 0, i=1,2,3$, the resulting theory violates causality and unitarity [4], [5]. Therefore, we shall consider only space-space noncommutativity with a choice of space coordinates such that $\theta^{12}=-\theta^{21} \equiv \theta$ with all other $\theta^{i j}$ 's vanishing. The commutation relations (II) preserve the translational symmetry but break the Lorentz invariance. With the choice $\theta^{12} \neq 0$ the Lorentz symmetry is broken down to the residual $S O(1,1) \times S O(2)$ symmetry formed by boost in 3-rd space direction and rotations in (1,2)-plane.

An axiomatic formulation of $\mathrm{NC}$ QFT, based on this residual space-time symmetry, was proposed in [6]. The resulting theory is effectively $(1+1)$ dimensional QFT in (0,3)-Minkowski plane supplemented by instantaneous interactions in $(1,2)$ Euclidean plane. The methods of usual (commutative) relativistic QFT are applied in (0,3)-Minkowski plane, whereas the role of NC coordinates $\hat{x}^{1}$ and $\hat{x}^{2}$ is strongly suppressed. This enables to investigate some general aspects which can be adapted to the residual space-time symmetry. 
However, this approach cannot be considered to be satisfactory: (i) There are no obvious reasons why the full relativistic symmetry should emerge in the commutative limit $\theta \rightarrow 0$, and (ii) there is no natural way how to introduce the spin and the classification of particles.

Recently, in [7] was proposed an alternative interpretation of commutation relations (11). Using the notion of twisted Poincaré symmetry (with all ten generators $P_{\mu}$ and $M_{\mu \nu}$ ) the interpretation of (11) was extended from Lie algebra framework to Hopf algebras: If in the usual (commutative) case, relativistic invariance means symmetry under the Poincaré transformations, in the noncommutative case relativistic invariance means symmetry under twisted Poincaré transformations. This "hidden" symmetry enables us to discuss all the aspects of relativistic QFT, which are not accessible within the residual symmetry approach. For example, it justifies the attempt to prove the spin-statistics theorem in [8] in Lagrangian formulation and in [9] within the axiomatic approach.

The main aim of this paper is to present a consistent frame for NC QFT with the relativistic invariance realized in terms of the twisted Poincaré symmetry proposed previously and to apply the developed techniques to the investigation and proof of the Haag theorem [10], with the statement: If a field at a certain time is related to a free one by a unitary transformation, as is the case in the interaction picture, then the field is inevitably free. This theorem concerns deep mathematical subtleties of systems possessing an infinite number of degrees of freedom, as is the case of QFT. It is an ultimate question to understand its background and nature also in the framework of NC QFT. Since the latter theories are nonlocal, one might think that the 
analog of Hagg's theorem may no more be valid due to the nature of $\mathrm{NC}$ field theories: while interacting fields are nonlocal, the free fields are local.

In Section 2 we shall first present the basic notions defining the twisted Poincaré symmetry, and then we perform the twist deformation of the QFT operator algebra generated by powers of quantum fields. Section 3 is devoted to the proof of Haag's theorem in the twisted Poincaré symmetry approach. Section 4 contains the concluding remarks.

\section{Twisted NC QFT}

Twisted Poincaré symmetry. First we briefly describe the twisted Poincaré Hopf-algebra $\mathcal{U}_{t}(\mathcal{P})$ proposed recently in [7] (for basic notions see [11]):

(a) The usual Poincaré algebra $\mathcal{P}$ is spanned by the generators of translations $P_{\mu}$ and Lorentz transformations $M_{\mu \nu}=-M_{\nu \mu}, \mu, \nu=0,1,2,3$, satisfying the usual commutation relations. Its enveloping algebra $\mathcal{U}(\mathcal{P})$ is a so called trivial Hopf-algebra with the co-product $\Delta(X)=X \otimes 1+1 \otimes X$.

(b) An essential object for us is the twisted Poincaré Hopf-algebra $\mathcal{U}_{t}(\mathcal{P})$. In $\mathcal{U}_{t}(\mathcal{P})$ the co-product is defined by $\Delta_{t}(X)=\mathcal{F} \Delta(X) \mathcal{F}^{-1}$ with the twist given as

$$
\mathcal{F}=e^{\frac{i}{2} \theta^{\mu \nu} P_{\mu} \otimes P_{\nu}}, P_{\mu}(X) \equiv\left[P_{\mu}, X\right] .
$$

Using $\theta_{\mu}{ }^{\rho}=\eta_{\mu \sigma} \theta^{\sigma \rho}$, equation (2) implies (see [7], [12]):

$$
\begin{gathered}
\Delta_{t}\left(P_{\mu}\right)=P_{\mu} \otimes 1+1 \otimes P_{\mu} \\
\Delta_{t}\left(M_{\mu \nu}\right)=M_{\mu \nu} \otimes 1+1 \otimes M_{\mu \nu} \\
-\frac{1}{2}\left(\theta_{\mu}{ }^{\rho} P_{\nu}-\theta_{\nu}{ }^{\rho} P_{\mu}\right) \otimes P_{\rho}+\frac{1}{2} P_{\rho} \otimes\left(\theta_{\mu}{ }^{\rho} P_{\nu}-\theta_{\nu}{ }^{\rho} P_{\mu}\right) .
\end{gathered}
$$


The antipode (the reverse) and co-unit are defined in $\mathcal{U}(\mathcal{P})$ and $\mathcal{U}_{t}(\mathcal{P})$ in the standard way; we shall not need them explicitly.

Having the representation of a Hopf-algebra in an associative algebra $\hat{\mathcal{A}}$ compatible with the co-product (Leibniz rule), the multiplication $m(\hat{a} \otimes \hat{b}) \equiv$ $\hat{a} \hat{b}$ in $\hat{\mathcal{A}}$ has to be changed after a twisting according to (2). In our particular case the twisted multiplication ( $\star$-product) reads [7]:

$$
\hat{a} \star \hat{b} \equiv m_{t}(\hat{a} \otimes \hat{b})=m\left(e^{-\frac{i}{2} \theta^{\mu \nu} \hat{P}_{\mu} \otimes \hat{P}_{\nu}} \hat{a} \otimes \hat{b}\right), \hat{P}_{\mu}(\hat{a}) \equiv\left[\hat{P}_{\mu}, \hat{a}\right],
$$

where $\hat{P}_{\mu}$ is the representation of $P_{\mu}$ in $\hat{\mathcal{A}}$, and similarly $\hat{M}_{\mu \nu}$ denotes the representation of $M_{\mu \nu}$.

Algebra of quantum fields. Below we shall apply this abstract concept to the algebra $\hat{\mathcal{A}}$ of operators in QFT Hilbert space $\mathcal{H}$ generated by products of fields $\hat{\phi}\left(x_{1}\right) \ldots \hat{\phi}\left(x_{n}\right)$, where $x_{1}, \ldots, x_{n}$, are space-time points in the Minkowski space $M$.

We shall assume that $\mathcal{H}$ carries a unitary representation of Poincaré algebra $\mathcal{P}$ with self-adjoint generators $\hat{P}_{\mu}$ and $\hat{M}_{\mu \nu}$. As usual we assume that $\hat{P}_{\mu}$ has the spectrum $V_{+}=\left\{p^{\mu} ; p^{0} \geq|\vec{p}|\right\}$. Furthermore, we assume that there is a unique $\mathcal{P}$-invariant vacuum state $|0\rangle \in \mathcal{H}: \hat{P}_{\mu}|0\rangle=\hat{M}_{\mu \nu}|0\rangle=0$, and that $|0\rangle$ is a cyclic vector in Hilbert space, i.e. $\mathcal{H}$ is spanned by vectors $\hat{\phi}\left(x_{1}\right) \ldots \hat{\phi}\left(x_{n}\right)|0\rangle, x_{1}, \ldots, x_{n} \in M$.

The operators $\hat{P}_{\mu}$ and $\hat{M}_{\mu \nu}$ act on fields in a standard way:

$$
\begin{gathered}
{\left[\hat{P}_{\mu}, \hat{\phi}(x)\right]=-\mathrm{i} \partial_{\mu} \hat{\phi}(x) \equiv\left(\mathcal{P}_{\mu} \hat{\phi}\right)(x),} \\
{\left[\hat{M}_{\mu \nu}, \hat{\phi}(x)\right]=\mathrm{i}\left(x_{\mu} \partial_{\nu}-x_{\nu} \partial_{\mu}\right) \hat{\phi}(x) \equiv\left(\mathcal{M}_{\mu \nu} \hat{\phi}\right)(x) .}
\end{gathered}
$$

We extend this action via commutators to any product of fields. For example, the Lorentz generator $\hat{M}_{\omega}=\frac{1}{2} \omega^{\mu \nu} \hat{M}_{\mu \nu}, \omega^{\mu \nu}=-\omega^{\nu \mu}$, acts on the product 
$\hat{\phi}(x) \hat{\phi}(y)$ as follows:

$$
\begin{aligned}
\mathcal{M}_{\mu \nu}(\hat{\phi}(x) \hat{\phi}(y)) & \equiv\left[\hat{M}_{\mu \nu}, \hat{\phi}(x) \hat{\phi}(y)\right]=\left[\hat{M}_{\mu \nu}, \hat{\phi}(x)\right] \hat{\phi}(y)+\hat{\phi}(x)\left[\hat{M}_{\mu \nu}, \hat{\phi}(y)\right] \\
& =\left(\mathcal{M}_{\mu \nu} \hat{\phi}\right)(x) \hat{\phi}(y)+\hat{\phi}(x)\left(\mathcal{M}_{\mu \nu} \hat{\phi}\right)(y)
\end{aligned}
$$

Equipped with this action, the algebra $\hat{\mathcal{A}}$ carries a representation of the trivial Hopf algebra $\mathcal{U}(\mathcal{P})$.

Deforming now the algebra $\hat{\mathcal{A}}$ by the twist (2), we arrive at the field operator algebra $\hat{\mathcal{A}}_{\star}$ carrying the representation of $\mathcal{U}_{t}(\mathcal{P})$ with the twisted operator product specified by (4) and (51):

$$
\hat{\phi}(x) \star \hat{\phi}(y)=m\left(e^{-\frac{\dot{i}}{2} \theta^{\mu \nu} \hat{P}_{\mu} \otimes \hat{P}_{\nu}} \hat{\phi}(x) \otimes \hat{\phi}(y)\right)=e^{\frac{i}{2} \theta^{\mu \nu} \partial_{x} \mu \partial_{y^{\nu}}} \hat{\phi}(x) \hat{\phi}(y)
$$

We would like to stress that although (7) looks like a Moyal product, there has been no noncommutativity of coordinates used. The $\star$-product is inherited from the twist of the operator product of quantum fields.

Locality condition. The locality condition is an independent axiom. It is the only place where the twist explicitly influences the field axioms, since at this point we are dealing with the products of fields.

Let us now investigate the transformation properties of star-product of fields $\hat{\phi}(x) \star \hat{\phi}(y)$ under the Lorentz transformation $\hat{M}_{\omega}=\frac{1}{2} \omega^{\mu \nu} \hat{M}_{\mu \nu}, \omega^{\mu \nu}=$ $-\omega^{\nu \mu}$. From (3) $-(15)$ it follows that the corresponding action of the Lorentz generator is given by:

$$
\begin{gathered}
\mathcal{M}_{\omega}^{t}(\hat{\phi}(x) \star \hat{\phi}(y)) \equiv m_{t}\left(\Delta_{t}\left(\hat{M}_{\omega}\right) \hat{\phi}(x) \otimes \hat{\phi}(y)\right) \\
=\left(\mathcal{M}_{\omega} \hat{\phi}\right)(x) \star \hat{\phi}(y)+\hat{\phi}(x) \star\left(\mathcal{M}_{\omega} \hat{\phi}\right)(y) \\
-\frac{1}{2} \theta^{\rho \sigma} \omega_{\rho}^{\nu}\left(\mathcal{P}_{\nu} \hat{\phi}\right)(x) \star\left(\mathcal{P}_{\sigma} \hat{\phi}\right)(y)-\frac{1}{2} \theta^{\rho \sigma} \omega_{\sigma}^{\nu}\left(\mathcal{P}_{\rho} \hat{\phi}\right)(x) \star\left(\mathcal{P}_{\nu} \hat{\phi}\right)(y)
\end{gathered}
$$


In spite of the $\theta$-corrections in the last line, the operators $\mathcal{M}_{\omega}^{t}$ satisfy the Lorentz algebra commutation relations:

$$
\left[\mathcal{M}_{\omega}^{t}, \mathcal{M}_{\omega^{\prime}}^{t}\right](\hat{\phi}(x) \star \hat{\phi}(y))=\mathrm{i} \mathcal{M}_{\omega \times \omega^{\prime}}^{t}(\hat{\phi}(x) \star \hat{\phi}(y))
$$

where $\left(\omega \times \omega^{\prime}\right)^{\mu \nu}=\omega^{\mu \sigma} \omega_{\sigma}^{\prime}{ }^{\nu}-\omega^{\mu \sigma} \omega_{\sigma}{ }^{\nu}$. This follows from the equation

$$
\mathcal{M}_{\omega}^{t}=e^{\frac{i}{2} \theta^{\mu \nu} \partial_{x} \mu \partial_{y^{\nu}}} \mathcal{M}_{\omega}^{t} e^{-\frac{i}{2} \theta^{\mu \nu} \partial_{x} \mu \partial_{y^{\nu}}},
$$

which simply reflects the fact that the twist in question influences the coproduct but not the basic algebraic structure itself.

Thus, we can exponentiate the action of Lorentz generators in a standard way, and obviously this is valid for translations too. In the Appendix it is shown that this guarantees the twisted Poincaré covariance of the $\star$-product of fields:

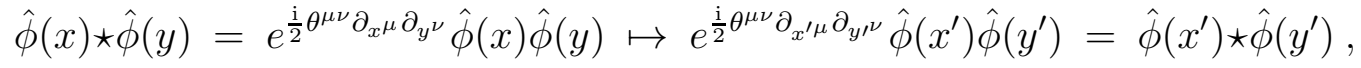

where $x^{\prime}=\Lambda x+a$ and $y^{\prime}=\Lambda y+a$ are the accordingly transformed space-time points. We stress that $\theta^{\mu \nu}$ is a twisted Poincaré invariant tensor: $\theta^{\mu \nu}=\theta^{\prime \mu \nu}$. This is natural and in full agreement with [7].

The covariance property (10) dictates the relativistic form of locality condition among $\star$-products of fields. We postulate it in the simplest form:

$$
(x-y)^{2}=\left(x^{0}-y^{0}\right)^{2}-(\vec{x}-\vec{y})^{2}<0 \Rightarrow[\hat{\phi}(x), \hat{\phi}(y)]_{\star}=0 .
$$

Note: In [13] was discussed, within NC context, a weaker locality condition in the form

$$
(x-y)^{2}<-l^{2} \Rightarrow[\hat{\phi}(x), \hat{\phi}(y)]_{\star}=0
$$


with $l^{2} \approx|\theta|$. In [14] it was proven that in a field theory satisfying translational invariance and spectral condition $p \in V_{+}$, the locality condition (12) implies (11). In our case, these requirements are indeed satisfied, thus supporting the use of the form (11) for the locality axiom.

\section{Haag's theorem}

Wightman functions. The NC Wightman functions are defined as the vacuum expectation values of multiple $\star$-products of fields [9]:

$$
\begin{aligned}
& W_{\star}\left(x_{1}, \ldots, x_{n}\right) \equiv\left\langle 0\left|\hat{\phi}\left(x_{1}\right) \star \ldots \star \hat{\phi}\left(x_{n}\right)\right| 0\right\rangle
\end{aligned}
$$

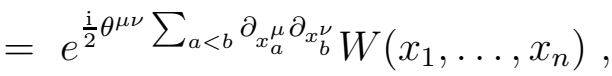

where $W\left(x_{1}, \ldots, x_{n}\right)=\left\langle 0\left|\hat{\phi}\left(x_{1}\right) \ldots \hat{\phi}\left(x_{n}\right)\right| 0\right\rangle$. Due to translational invariance, the $\theta$-dependent exponent in (131) can be omitted for the 2-point NC Wightman function: $W_{\star}(x, y)=W(x, y)=\mathcal{W}(x-y)$. However, it cannot be omitted for the higher NC Wightman functions.

The NC Wightman functions defined above satisfy the twisted Poincaré covariance condition and the twisted Poincaré locality condition:

$$
\begin{gathered}
W_{\star}\left(x_{1}, \ldots, x_{n}\right) \mapsto W_{\star}\left(\Lambda x_{1}+a, \ldots, \Lambda x_{n}+a\right), \\
(x-y)^{2}<0 \Rightarrow W_{\star}(\ldots, x, y, \ldots)=W_{\star}(\ldots, y, x, \ldots),
\end{gathered}
$$

together with the usual positivity axiom (the positivity of the norm in $\mathcal{H}$ ).

This enables to formulate and discuss various fundamental NC QFT theorems along the lines similar to the standard ones. The approach based on twisted Poincaré symmetry can offer a firm foundation for the proofs of $\mathrm{NC}$ 
analogs of the CPT theorem and spin-statistics theorem in the axiomatic approach.

Haag's theorem. As an interesting application of the twisted Poincaré formalism we prove the Haag theorem. The theorem was originally formulated in [10]; its variations and extensions were presented in [15] (for a detailed discussion, see [16]). Below we briefly discuss a sequence of theorems leading, in the twisted Poincaré context, to the Haag theorem. The first theorem we need can be formulated as follows:

Theorem 1: Let the 2-point function $\langle 0|\hat{\phi}(x) \star \hat{\phi}(y)| 0\rangle$ of an arbitrary scalar field $\hat{\phi}(x)$ coincide with the 2-point function of a free field of mass $m>0,-\mathrm{i} \Delta(x-y ; m)$, satisfying the Klein-Gordon equation, so that

$$
\left(\partial_{x}^{2}+m^{2}\right)\langle 0|\hat{\phi}(x) \star \hat{\phi}(y)| 0\rangle=-\mathrm{i}\left(\partial_{x}^{2}+m^{2}\right) \Delta(x-y ; m)=0 .
$$

Then the interaction current vanish: $\hat{J}(x) \equiv\left(\partial_{x}^{2}+m^{2}\right) \hat{\phi}(x)=0$, i.e. the theory is free.

Proof: The first part of the proof is identical to the usual one [15]. Considering an arbitrary test function $F(x)$ we shall prove that the state $|\psi\rangle \equiv \int d x F(x) \hat{J}(x)|0\rangle=\int d x F(x)\left(\partial_{x}^{2}+m^{2}\right) \hat{\phi}(x)|0\rangle$ vanishes. Omitting the *-product in (15) we obtain

$$
\langle\psi \mid \psi\rangle=\int d x d y \bar{F}(x) F(y)\left(\partial_{x}^{2}+m^{2}\right)\left(\partial_{y}^{2}+m^{2}\right)\langle 0|\hat{\phi}(x) \hat{\phi}(y)| 0\rangle=0 .
$$

Since $F(x)$ is arbitrary, the current $\hat{J}(x)$ annihilates the vacuum state: $\hat{J}(x)|0\rangle$ $=0$.

The nontrivial part of the theorem is based on the existence of $\mathrm{NC}$ analogs of Jost-points for the Wightman functions $W_{\star}\left(x_{1}, \ldots, x_{n-1}, x_{n}\right)=$ $\left\langle 0\left|\hat{\phi}\left(x_{1}\right) \star \ldots \star \hat{\phi}\left(x_{n-1}\right) \star \hat{\phi}\left(x_{n}\right)\right| 0\right\rangle$ which are boundary values of a holomorphic 
function $W_{\star}\left(z_{1}, \ldots, z_{n-1}, z_{n}\right)$ of complex variables $z_{1}, \ldots, z_{n-1}, z_{n}$ in a proper domain (the extended tube) containing real Jost points $\left(r_{1}, \ldots, r_{n-1}, r_{n}\right)$, satisfying $\left(r_{k}-r_{l}\right)^{2}<0$, for all $k>l$. Due to the locality condition (14), we can permute the fields evaluated at Jost points: $W_{\star}\left(r_{1}, \ldots, r_{n-1}, r_{n}\right)=$ $W_{\star}\left(r_{1}, \ldots, r_{n}, \ldots, r_{n-1}\right)$. Performing now in this equation the analytical continuation back to $x_{1} \ldots, x_{n-1}, x_{n}$, and acting by $\left(\partial_{x_{n}}^{2}+m^{2}\right)$ we obtain:

$$
\left\langle 0\left|\hat{\phi}\left(x_{1}\right) \star \ldots \star \hat{\phi}\left(x_{n-1}\right) \star \hat{J}\left(x_{n}\right)\right| 0\right\rangle=\left\langle 0\left|\hat{\phi}\left(x_{1}\right) \star \ldots \star \hat{J}\left(x_{n}\right) \star \ldots \star \hat{\phi}\left(x_{n-1}\right)\right| 0\right\rangle .
$$

Since $\hat{J}(x)|0\rangle=0$, the l.h.s. of (16) is zero. The r.h.s. represents, due to the completeness axiom, an arbitrary matrix element of the interaction current. Consequently, $\hat{J}(x)=0$, i.e. the theory is free.

To prove the Haag theorem we need a simple theorem dealing with fixed time fields. Its formulation and proof is similar to the usual one (see [16]).

Theorem 2: Let $\hat{\phi}_{1}(\vec{x}, t)$ and $\hat{\phi}_{2}(\vec{x}, t)$ be two irreducible scalar fields at a fixed time $t$ defined respectively in Hilbert spaces $\mathcal{H}_{1}$ and $\mathcal{H}_{2}$ in which there are two continuous unitary representations of the Euclidean group E(3):

$$
U_{j}(\vec{a}, R) \hat{\phi}_{j}(\vec{x}, t) U_{j}^{\dagger}(\vec{a}, R)=\hat{\phi}_{j}(R \vec{x}+\vec{a}, t), j=1,2 .
$$

We assume that: (i) The representations possess unique invariant vacuum states $|0\rangle_{j}: U_{j}(\vec{a}, R)|0\rangle_{j}=|0\rangle_{j}, j=1,2$, and (ii) there exists a unitary operator $V: \mathcal{H}_{1} \rightarrow \mathcal{H}_{2}$ such that at time $t: \hat{\phi}_{2}(\vec{x}, t)=V \hat{\phi}_{1}(\vec{x}, t) V^{\dagger}$.

Then $U_{2}(\vec{a}, R)=V U_{1}(\vec{a}, R) V^{\dagger},|0\rangle_{2}=e^{\mathrm{i} \alpha} V|0\rangle_{1}, \alpha \in \mathbf{R}^{1}$.

Note: The group $E(3)$ is a subgroup of the Poincaré group generated by rotations and translations in $\mathbf{R}^{3}$. This induces the representation of the twisted Euclidean Hopf-algebra by twist (2) in the operator algebra generated 
by field operators at the fixed time $t$. It is a Hopf subalgebra of $\hat{\mathcal{A}}_{\star}$ - this is the place where the conditions $\theta^{0 i}=0, i=1,2,3$, become essential.

Corollary: The equal time vacuum expectation values of both theories coincide: ${ }_{1}\left\langle 0\left|\hat{\phi}_{1}\left(\vec{x}_{1}, t\right) \star \ldots \star \hat{\phi}_{1}\left(\vec{x}_{n}, t\right)\right| 0\right\rangle_{1}={ }_{2}\left\langle 0\left|\hat{\phi}_{2}\left(\vec{x}_{1}, t\right) \star \ldots \star \hat{\phi}_{2}\left(\vec{x}_{n}, t\right)\right| 0\right\rangle_{2}$.

Theorem 1 and the Corollary provide us with the proof of Haag's theorem:

Theorem 3 (Haag): Suppose $\hat{\phi}_{1}(x)$ is a free hermitian scalar field of mass $m>0$, and $\hat{\phi}_{2}(x)$ is a scalar field satisfying the NC QFT axioms given above. Suppose further that the fields $\hat{\phi}_{j}(x),\left(\partial_{t} \hat{\phi}\right)_{j}(x), j=1,2$, satisfy the hypothesis of Theorem 2. Then $\hat{\phi}_{2}(x)$ is a free field of mass $\mathrm{m}$.

Proof: Any two space-like separated points $x$ and $y$ can be brought by a Lorentz transformation to equal-time plane: $x=(\vec{x}, t)$ and $y=(\vec{y}, t)$. By Corollary we have ${ }_{2}\left\langle 0\left|\hat{\phi}_{2}(\vec{x}, t) \star \hat{\phi}_{2}(\vec{y}, t)\right| 0\right\rangle_{2}=-\mathrm{i} \Delta(\vec{x}-\vec{y}, 0 ; m)$. Using the standard analytic continuation argument and the twisted relativistic covariance of $\hat{\phi}_{2}(x) \star \hat{\phi}_{2}(y)$ we obtain: ${ }_{2}\left\langle 0\left|\hat{\phi}_{2}(x) \star \hat{\phi}_{2}(y)\right| 0\right\rangle_{2}=-\mathrm{i} \Delta(x-y ; m)$. The Haag's theorem is then a direct consequence of Theorem 1.

\section{Concluding remarks}

The approach presented above is based on the twisted Poincaré symmetry (with all 10 generators) which states that: while symmetry under the usual Lorentz transformations guarantees the relativistic invariance of a theory, in the $\mathrm{NC} \mathrm{QFT}$ the concept of relativistic invariance, however, should be replaced by the requirement of invariance of the theory under the twisted Poincaré transformations. The latter allows us to formulate and discuss all fundamental issues of relativistic QFT within NC context. Because of 
the content of its representations, the twisted Poincaré algebra offers a firm framework for the proofs of the $\mathrm{NC}$ version of CPT and the spin-statistics theorems 9], among other results obtained in the literature so far, and justifies in particular the results obtained in [8] within Lagrangian formulation. The Haag theorem also belongs to that class of exact (not perturbative) results.

Acknowledgements. We are grateful to R. Haag, P. Kulish and K. Nishijima for valuable discussions and remarks. The financial support of the Academy of Finland under the Projects No. 54023 and 104368 is greatly acknowledged. P.P.'s work was partially supported by project VEGA No. 1/025/03.

\section{Appendix}

Let us consider an infinitesimal Lorentz transformation $\hat{\phi}(x) \star \hat{\phi}(y) \mapsto$ $\left(1+\mathrm{i} \mathcal{M}_{\omega}^{t}\right)(\hat{\phi}(x) \star \hat{\phi}(y))$ generated by $\hat{M}_{\omega}=\frac{1}{2} \omega^{\mu \nu} \hat{M}_{\mu \nu}, \omega$ - infinitesimal.

According to the definition (8) we have:

$$
\begin{gathered}
\left(1+\mathrm{i} \mathcal{M}_{\omega}^{t}\right)(\hat{\phi}(x) \star \hat{\phi}(y))=m_{t}\left\{\left[1+\mathrm{i} \Delta_{t}\left(\hat{M}_{\omega}\right)\right][\hat{\phi}(x) \otimes \hat{\phi}(y)]\right\} \\
=m\left\{e ^ { - \frac { \mathrm { i } } { 2 } \theta ^ { \rho \sigma } \mathcal { P } _ { \rho } \otimes \mathcal { P } _ { \sigma } } \left[\hat{\phi}(x) \otimes \hat{\phi}(y)+\mathrm{i}\left(\mathcal{M}_{\omega} \hat{\phi}\right)(x) \otimes \hat{\phi}(y)+\mathrm{i} \hat{\phi}(x) \otimes\left(\mathcal{M}_{\omega} \hat{\phi}\right) \hat{\phi}(y)\right.\right. \\
\left.\left.-\frac{\mathrm{i}}{2} \theta^{\rho \sigma} \omega_{\rho}^{\nu}\left(\mathcal{P}_{\nu} \hat{\phi}\right)(x) \otimes\left(\mathcal{P}_{\sigma} \hat{\phi}\right)(y)-\frac{\mathrm{i}}{2} \theta^{\rho \sigma} \omega_{\sigma}^{\nu}\left(\mathcal{P}_{\rho} \hat{\phi}\right)(x) \otimes\left(\mathcal{P}_{\nu} \hat{\phi}\right)(y)\right]\right\} .
\end{gathered}
$$

Up to terms linear in $\omega$ this can be rewritten as

$$
\begin{gathered}
\left(1+\mathrm{i} \mathcal{M}_{\omega}^{t}\right)(\hat{\phi}(x) \star \hat{\phi}(y)) \doteq m\left\{e ^ { - \frac { \mathrm { i } } { 2 } \theta ^ { \rho \sigma } \mathcal { P } _ { \rho } \otimes \mathcal { P } _ { \sigma } } \left[\hat{\phi}\left(x^{\prime}\right) \otimes \hat{\phi}\left(y^{\prime}\right)\right.\right. \\
\left.\left.-\frac{\mathrm{i}}{2} \theta^{\rho \sigma} \omega_{\rho}^{\nu}\left(\mathcal{P}_{\nu} \hat{\phi}\right)\left(x^{\prime}\right) \otimes\left(\mathcal{P}_{\sigma} \hat{\phi}\right)\left(y^{\prime}\right)-\frac{\mathrm{i}}{2} \theta^{\rho \sigma} \omega_{\sigma}^{\nu}\left(\mathcal{P}_{\rho} \hat{\phi}\right)\left(x^{\prime}\right) \otimes\left(\mathcal{P}_{\nu} \hat{\phi}\right)\left(y^{\prime}\right)\right]\right\}
\end{gathered}
$$




$$
\begin{gathered}
\doteq m\left\{e^{-\frac{i}{2} \theta^{\rho \sigma}\left(\mathcal{P}_{\rho}+\omega_{\rho}{ }^{\nu} \mathcal{P}_{\nu}\right) \otimes\left(\mathcal{P}_{\sigma}+\omega_{\sigma}{ }^{\nu} \mathcal{P}_{\nu}\right)}\left(\hat{\phi}\left(x^{\prime}\right) \otimes \hat{\phi}\left(y^{\prime}\right)\right)\right\} \\
=e^{\frac{i}{2} \theta^{\rho \sigma} \partial_{x^{\prime \rho}} \partial_{y^{\prime}}} \hat{\phi}\left(x^{\prime}\right) \hat{\phi}\left(y^{\prime}\right)=\hat{\phi}\left(x^{\prime}\right) \star \hat{\phi}\left(y^{\prime}\right) .
\end{gathered}
$$

The symbol $\doteq$ denotes an equality up to first order in $\omega ; x^{\rho}=x^{\rho}-\omega_{\mu}{ }^{\rho} x^{\mu}$ and $y^{\prime \sigma}=y^{\sigma}-\omega_{\mu}^{\sigma} y^{\mu}$ are just the Lorentz transformed space-time points.

This proves the covariance relation (10) for infinitesimal Lorentz transformation. For a finite transformation, (10) is recovered by a usual exponentiation. The inclusion of translations is trivial. We point out that the covariance relation can be extended to multiple products of field.

\section{References}

[1] H. S. Snyder, Phys. Rev. 71 (1947) 38.

[2] S. Doplicher, K. Fredenhagen and J. E. Roberts, Commun. Math. Phys. $172(1995) 187$.

[3] N. Seiberg and E. Witten, JHEP 9909 (1999) 032.

[4] N. Seiberg, L. Susskind and N. Toumbas, JHEP 0006 (2000) 044, hep-th/0005015 L. Álvarez-Gaumé and J. L. F. Barbon, Int. J. Mod. Phys. A 16 (2001) 1123, hep-th/0006209.

[5] J. Gomis and T. Mehen, Nucl. Phys. B 591 (2000) 265, hep-th/0005129

[6] L. Álvarez-Gaumé and M. A. Vázquez-Mozo, Nucl. Phys. B 668 (2003) 293, hep-th/0305093. 
[7] M. Chaichian, P. Kulish, K. Nishijima and A. Tureanu, On a LorentzInvariant Interpretation of Quantum Field Theory on Noncommutative Noncommutative Space-Time, hep-th/0408069.

[8] M. Chaichian, K. Nishijima and A. Tureanu, Phys. Lett. B 568 (2003) 146, hep-th/0209008.

[9] M. Chaichian, M. N. Mnatsakanova, K. Nishijima, A. Tureanu and Yu. S. Vernov, Towards an Axiomatic Formulation of Noncommutative Quantum Field Theory, hep-th/0402212.

[10] R. Haag, Dan. Mat. Fys. Medd. 29 No. 12 (1955).

[11] M. Chaichian and A. Demichev Quantum Groups, World Scientific, Singapore, 1996; V. Chari and A. Pressley, A Guide to Quantum Groups, Cambridge University Press, Cambridge, 1994.

[12] J. Wess, Deformed Coordinate Spaces Derivatives, hep-th/0408080.

[13] M. Chaichian and A. Tureanu, Jost-Lehman-Dyson Representation and Froissart-Martin Bound in Quantum Field Theory on Noncommutative Space-Time, hep-th/0403032.

[14] V. S. Vladimirov, Sov. Math. Dokl. 1 (1960)1039; Methods of the Functions of Several Complex Variables, Cambridge, Massachusetts, MIT Press, 1966; D. Ya. Petrina, Ukr. Mat. Zh. 13 No. 4 (1961) 109 (in Russian); A. S. Wightman, J. Indian Math. Soc. 24 (1960-61) 625.

[15] P. G. Federbush and K. A. Johnson, Phys. Rev. 120 (1960) 1926; R. Jost, Properties of Wightman functions, in Lectures on Field Theory and 
Many-Body Problem, E. R. Caianiello (ed.), Academic Press, New York, 1961; O. W. Greenberg, Phys. Rev. 115 (1959) 706.

[16] R. F. Streater and A. S. Wightman, PCT, Spin \& Statistics, and All That, W. A. Benjamin, Inc., New York, 1964; N. N. Bogoliubov, A. A. Logunov and I. T. Todorov, Introduction to Axiomatic Quantum Field Theory, W. A. Benjamin, Inc., New York, 1975. 